\title{
Experiencia de aprendizaje con tecnologías digitales y su influencia en la competencia científica de estudiantes de secundaria
}

\author{
Claudia Vela-Acero \\ Colegio Veintiún Ángeles IED. Colombia. \\ claudia_acero07@yahoo.es \\ Rocío Jiménez-Cortés \\ Universidad de Sevilla. España. \\ rjimenez@us.es
}

Recibido: $17 / 10 / 2020$

Aceptado: $14 / 4 / 2021$

Publicado: 23/9/2021

\section{Resumen}

Estudios previos relacionan el uso educativo de las tecnologías digitales con la alfabetización científica, pero hay escasez de análisis que se centren en el impacto de la experiencia de aprendizaje con tecnologías en la competencia científica. Además, en relación con el género, la literatura científica es inconsistente. La investigación que presentamos es cuantitativa y sigue un método descriptivo con diseños comparativo y predictivo. Se busca la influencia de la experiencia de aprendizaje con tecnologías digitales en la competencia científica (autopercibida y observada) en las ciencias naturales de 160 estudiantes ( 80 chicos y 80 chicas) de educación básica secundaria en Bogotá (Colombia). Los hallazgos muestran que no existen diferencias significativas según género en la competencia científica, y la experiencia de aprendizaje con tecnologías digitales predice la competencia científica autopercibida. Los resultados se discuten con estudios recientes en torno a la formación científica mediada por tecnologías.

Palabras clave: estudios de género; alfabetización científica; educación secundaria; alfabetización tecnológica; experiencia de aprendizaje

Resum. Experiència d'aprenentatge amb tecnologies digitals i influència en la competència cientifica d'estudiants de secundària

Estudis previs relacionen l'ús educatiu de les tecnologies digitals amb l'alfabetització científica, però hi ha molt pocs estudis que se centrin en l'impacte de l'experiència d'aprenentatge amb tecnologies a la competència científica. A més, en relació amb el gènere, la literatura científica és inconsistent. La investigació que presentem és quantitativa i segueix un mètode descriptiu amb dissenys comparatiu i predictiu. Es busca la influència de l'experiència d'aprenentatge amb tecnologies digitals en la competència científica (autopercebuda i observada) en les ciències naturals de 160 estudiants ( 80 nois i 80 noies) d'educació bàsica secundària a Bogotà (Colòmbia). Les troballes mostren que no hi ha diferències significatives entre els gèneres en la competència científica, i l'experiència 
d'aprenentatge amb tecnologies digitals prediu la competència científica autopercebuda. Els resultats es discuteixen amb estudis recents entorn d'una formació científica mediada per tecnologies.

Paraules clau: estudis de gènere; alfabetització científica; educació secundària; alfabetització tecnològica; experiència d'aprenentatge

Abstract. Learning experience with digital technologies and its influence on the scientific competence of high school students

Previous studies link the educational use of digital technologies with scientific literacy, but there is a shortage of studies that focus on the impact of the learning experience with technologies on scientific competence. Furthermore, in relation to gender, the scientific literature is inconsistent. The research we present is quantitative and follows a descriptive method with comparative and predictive designs. We aim to determine the influence of a learning experience with digital technologies on scientific competence (self-perceived and observed) in the natural sciences among 160 students ( 80 boys and 80 girls) in basic secondary education in Bogotá, Colombia. The findings show that there are no significant differences according to gender in scientific competence and that the learning experience with digital technologies predicts self-perceived scientific competence. The results are discussed with recent studies on scientific training mediated by technologies.

Keywords: gender studies; scientific literacy; technological literacy; secondary education; experiential learning

\author{
Sumario \\ 1. Introducción 4. Resultados \\ 2. Objetivos 5. Discusión de resultados y conclusiones \\ 3. Metodología Referencias bibliográficas
}

\title{
1. Introducción
}

El uso de tecnologías digitales en la formación científica puede resultar un valor añadido para el desarrollo de competencias científicas en educación secundaria, pero hay escasez de estudios que nos permitan asegurarlo. Como afirman Hillmayr, Ziernwald, Reinhold, Hofer y Reiss (2020), el impacto del uso de herramientas digitales aún no está completamente claro. Trabajos actuales como el de Neiman (2017) muestran que la implementación de tecnologías digitales permite que los estudiantes puedan comparar, analizar, construir, integrar contenidos y capacidades para recombinar y enlazar conceptos. Además, estimulan procesos cognitivos y significación de aprendizajes. Una de las habilidades más significativas es la participación en línea sobre cuestiones sociocientíficas. Según Tsai (2018), la socialización de eventos controvertidos permite a los estudiantes utilizar conocimiento científico en la argumentación, lo que mejora sus habilidades de explicación y razonamiento investigador basado en evidencias y habilidades de pensamiento crítico fundamentales para ser un ciudadano científicamente alfabetizado. Por ello, el uso de las tecnolo- 
gías digitales no solo incide en el aprendizaje individual, sino, además, en el aprendizaje colectivo, al compartir y/o construir conocimiento o al plantear alternativas de solución a problemáticas del entorno, a través de foros virtuales o de redes sociales. Rincón (2015, p. 94) afirma que en el marco de las tecnologías digitales más populares en la enseñanza de las ciencias se encuentran las simulaciones. Estas se convierten en unas herramientas versátiles que no solo llaman la atención e incrementan la motivación del estudiante, sino que también permiten que el profesorado, por medio de estas, incentive al colectivo estudiantil a proponer hipótesis, explicaciones y predicciones haciendo uso del lenguaje científico. Estas habilidades resultan básicas para desarrollar las competencias científicas.

Estudios como el de Luu y Freeman (2011) apuntan a que el uso de las tecnologías digitales con fines educativos, a través de una gran variedad de software y aplicaciones de Internet accesibles para los estudiantes, puede ser potencialmente beneficioso para el logro en materias relacionadas con la ciencia. Sin embargo, no hay análisis concluyentes en la línea. Así, la investigación de Hu, Gong, Lai y Leung (2018), que aborda el uso educativo de las tecnologías digitales y el rendimiento de los estudiantes, muestra que correlaciona negativamente. Otros más recientes, como el de Odell, Galovan y Cutumisu (2020), evalúan el impacto del uso, la disponibilidad y la comodidad de las TIC en los puntajes en ciencias de los estudiantes. Este análisis comparativo entre Bulgaria y Finlandia revela que los estudiantes que se sentían más cómodos con las TIC se desempeñaron mejor en ciencias. Pero variables como la disponibilidad y el uso de TIC se asociaban con un rendimiento bajo en ciencias. A pesar de ello, estas investigaciones concluyen que las tecnologías deben emplearse cuidadosamente en entornos educativos y deben ir vinculadas a otras acciones para mejorar la calidad de su integración. Otros trabajos, como el de Romero y Quesada (2014), recogen ejemplos que pueden resultar especialmente ilustrativos en torno a la influencia de la tecnología en el aprendizaje de las ciencias. También, el metaanálisis realizado por Hillmayr et al. (2020) integra trabajos que comparan los resultados de aprendizaje de los estudiantes que utilizan herramientas digitales con los de un grupo de control a los que se enseña sin empleo de herramientas digitales. En general, el uso de estas tuvo un efecto positivo en los resultados de aprendizaje en ciencias de los alumnos. Específicamente, el estudio de Winarni, Hambali y Purwandari (2020) muestra que existe una influencia entre aprender con las TIC y la competencia científica de los estudiantes. También el de Petko, Cantieni y Prasse (2017) evidencia que las actitudes positivas hacia la tecnología educativa están asociadas con puntajes más altos en las pruebas de competencia científica en la gran mayoría de los países. Y además concluye que, dado que es probable que las actitudes positivas sean el resultado de experiencias positivas, parece razonable que la calidad en lugar de la cantidad de uso de tecnología educativa sea la clave. Esto plantea la relevancia de examinar la experiencia de aprendizaje con tecnologías.

Los informes internacionales demuestran los bajos resultados obtenidos en ciencias naturales en secundaria. Así, se observa que cerca del $20 \%$ de los 
estudiantes de los países de la OCDE (2015) rinde por debajo del nivel 2, considerado el umbral básico de competencias científicas y debiendo alcanzar este nivel al concluir la educación obligatoria. Estos resultados señalan las dificultades que los alumnos presentan para la alfabetización científica y el desarrollo de aprendizajes significativos. Al desagregar los resultados obtenidos en Bogotá según el género de los estudiantes, es posible observar que los niños muestran un mejor desempeño en el área de ciencias en el 2015, al obtener 463 puntos, y las niñas, 452 (MEN, 2017, p. 24). Sin embargo, análisis actuales no se muestran consistentes. O bien indican que no hay diferencias de género en la alfabetización científica (Hardinata, Putri y Permanasari, 2019) o incluso los resultados son más favorables a las chicas (Kristyasari, Yamtinah, Utomo e Indriyanti, 2018; Naganuma, 2017). Esto puede estar indicando cambios en las brechas de género o en la medida de los desempeños en el ámbito de la competencia científica.

En cuanto a las tecnologías digitales y el género, la investigación ha puesto de manifiesto de forma más clarificadora las brechas existentes en la capacitación en tecnologías entre chicos y chicas (Voyer y Voyer, 2014). Así, Ramírez y Dávila (2017) encontraron que un elevado porcentaje de niñas experimentan con mayor frecuencia entusiasmo y confianza en el aprendizaje de contenidos como el proceso de resolución de problemas tecnológicos y materiales de uso técnico, mientras que los niños experimentan más preocupación, nerviosismo y aburrimiento. Con respecto al aprendizaje de contenidos sobre expresión y comunicación técnica, las niñas experimentan más nerviosismo, preocupación y miedo, mientras que los niños experimentan alegría, confianza, entusiasmo y diversión. Pero estudios muy actuales que no se basan en la alfabetización en TIC autoinformada sugieren que la brecha de género en las TIC puede no ser tan grave como se había afirmado (Siddiq y Scherer, 2019).

La experiencia de aprendizaje con tecnologías digitales es un constructo novedoso en la literatura nacional e internacional. Si bien hay estudios (como el PISA) que se han ocupado de conceptos parciales como conectividad en el colegio, número de ordenadores en función del tamaño del centro, entre otros, desde los cuales inferir una experiencia de uso, lo cierto es que, específicamente, no ha sido abordado en el sentido más abarcador que planteamos. A nivel internacional, el constructo más próximo que recoge la literatura científica y de acuerdo también con el planteamiento que hace PISA es el de «experience computering» en relación con la alfabetización científica (Hu et al., 2018).

En nuestro trabajo la experiencia de aprendizaje con tecnologías digitales se encuadra en un enfoque constructivista y en el marco de la teoría del aprendizaje experiencial (Kolb y Kolb, 2017). De ahí que el concepto planteado por la teoría constructivista como "experiential learning» (Kolb y Kolb, 2017) enfatice la importancia de la experiencia en la construcción del conocimiento. Autores relevantes que han planteado contribuciones en los últimos cien años en torno a esta teoría han sido, entre otros, John Dewey y Mary Parker Follett. Según Kolb y Kolb (2017), el término aprendizaje experiencial es empleado por muchos autores para referirse a ejercicios y juegos utilizados para involu- 
crar a los estudiantes en el proceso de aprendizaje. En la definición del constructo que planteamos incorporamos indicadores como la participación y el trabajo colaborativo haciendo uso de herramientas tecnológicas como chat o Google Drive o de interacción en foros virtuales, simuladores, tutoriales interactivos y cuestionarios en línea, así como realización de actividades grupales por Internet para desarrollar investigaciones. De ahí que se proponga este constructo desde dos perspectivas para el trabajo que presentamos. Por un lado, la que plantea la inmersión del estudiante en experiencias de aprendizaje auténtico que permita considerar que ha aprendido de forma previa desde la mediación de las tecnologías digitales y, por otro, la que contempla una serie de aspectos contextuales que determinan la experiencia de uso, como, por ejemplo, utilizar las tecnologías en casa para completar lo visto en clase o la disponibilidad de ordenadores en el centro educativo a criterio del estudiante. $\mathrm{Hu}$ et al. (2018) incorporan algunos aspectos relacionados con la experiencia de aprendizaje con tecnologías digitales, como pueden ser la conectividad o el número de ordenadores en función del tamaño del centro. Estos aspectos también los hemos incorporado como parte del constructo global de experiencia de aprendizaje con tecnologías digitales desde la percepción del estudiante, en nuestro caso.

Con respecto a las competencias científicas en Colombia, el Ministerio de Educación Nacional (MEN) establece, en la guía sobre los estándares básicos de competencias en ciencias, que la formación académica en esta área debe

[...] contribuir a la consolidación de ciudadanos y ciudadanas capaces de asombrarse, observar y analizar lo que acontece a su alrededor y en su propio ser, formularse preguntas, buscar explicaciones y recoger información, detenerse en sus hallazgos, analizarlos, establecer relaciones, hacerse nuevas preguntas y aventurar nuevas comprensiones, compartir y debatir con otros sus inquietudes, sus maneras de proceder, sus nuevas visiones del mundo, buscar soluciones a problemas determinados y hacer uso ético de los conocimientos científicos, todo lo cual aplica por igual para fenómenos tanto naturales como sociales. (MEN, 2004)

La OCDE (2015) define la competencia científica como la capacidad que tiene un ciudadano reflexivo para involucrarse en cuestiones relacionadas con la ciencia y con las ideas de la ciencia. En este trabajo empleamos la base conceptual de los indicadores planteados en estos documentos de referencia para abordar la medida de la competencia científica, y lo hacemos de acuerdo también con la literatura científica internacional (Parno et al., 2020; Rosa et al., 2018).

La conceptualización del Programa de Evaluación Internacional de Estudiantes (PISA) es un referente importante, ya que define componentes de la competencia científica, aunque no universitaria. Este programa organizado por la Organización para la Cooperación y el Desarrollo Económicos (OCDE) entiende la competencia científica como la habilidad para comprometerse con cuestiones relacionadas con la ciencia y con las ideas científicas. Este programa 
enfatiza que la formación científica de estudiantes de secundaria incluye varias capacidades: 1. Explicar fenómenos científicamente (esta dimensión incluye el reconocimiento de preguntas que sería posible investigar y de las características clave de una investigación científica), 2. Evaluar y diseñar investigaciones científicas (necesaria para evaluar de manera crítica los informes de investigación) y 3 . Interpretar científicamente datos y evidencias (esta dimensión incluye el acceso a la información científica y la construcción de argumentos y conclusiones basadas en evidencia científica). Estas capacidades muestran aspectos clave de la competencia científica considerados en este trabajo. En este sentido, se plantea la competencia científica, tanto autopercibida (en tanto que los estudiantes y las estudiantes son quienes indican y valoran su capacidad para explicar fenómenos científicamente, para evaluar y diseñar investigaciones científicas y para interpretar científicamente datos y evidencias) como observada (es decir, midiendo el desempeño y el desarrollo de esas capacidades en tareas propuestas y en resolución de casos específicos).

\section{Objetivos}

El estudio pretende indagar en la experiencia de aprendizaje con tecnologías digitales y en las competencias científicas (autopercibidas y observadas) de un grupo de estudiantes colombianos de secundaria según género. Este objetivo general se concreta en cuatro objetivos específicos:

- Conocer la experiencia de aprendizaje con tecnologías digitales del alumnado participante según género.

- Conocer las competencias científicas autopercibidas del alumnado participante según género.

- Conocer las competencias científicas observadas a partir del desempeño del alumnado en actividades de ciencias naturales relacionadas con contenidos como entorno vivo, entorno físico y ciencia, tecnología y sociedad.

- Establecer si la experiencia de aprendizaje con tecnologías digitales o las competencias científicas observadas en el desempeño de actividades de ciencias naturales se muestran predictoras de las competencias científicas autopercibidas en el alumnado de secundaria.

\section{Metodología}

El estudio sigue una metodología cuantitativa, concretamente un método descriptivo tipo encuesta. Se aplica un diseño comparativo para establecer diferencias según género, tanto en las competencias científicas como en la experiencia de aprendizaje con tecnologías digitales y predictivo de la competencia científica autopercibida. 


\subsection{Muestreo y participantes}

Se emplea un procedimiento de muestreo incidental seleccionando cuatro grupos naturales de estudiantes de octavo y noveno grado de un colegio oficial de Bogotá ubicado en la localidad de Suba. En total participan 160 estudiantes (80 chicos y 80 chicas) de edades comprendidas entre los 12 y los 17 años. Los estudiantes, por su estado socioeconómico, se ubican en estratos 1 al 3, provienen de los barrios cercanos a la institución, la mayoría cuentan con un computador de escritorio en su casa, muchos conviven con el papá o la mamá únicamente.

\subsection{Instrumento y medidas}

El cuestionario que se emplea incorpora variables de perfil como género, edad o curso. También incorpora medidas específicas como:

- Experiencia de aprendizaje con tecnologías digitales. Medida adaptada del cuestionario de Pérez Valverde (2015). Concretamente, se plantean a modo de escala tipo Likert un conjunto de sentencias (10 ítems) que abordan la experiencia percibida de aprendizaje con tecnologías digitales: 1 . Manejo la computadora y navego por Internet sin muchas dificultades, 2. He cambiado el aula por la sala de informática para recibir clases diferentes de informática alguna vez durante el año escolar, 3 . Hay suficientes computadoras en el colegio para aprovechar las TIC en el aula, 4. Estoy satisfecho con la conectividad actual del colegio, 5 . Uso las TIC en casa para complementar lo visto en las clases, 6. He usado o me han sugerido vídeos, blogs, páginas web o plataformas para apoyar el aprendizaje, 7. He participado y he trabajado colaborativamente haciendo uso de herramientas tecnológicas como chat o Google Drive, 8 . He interactuado en foros virtuales, simuladores, tutoriales interactivos y cuestionarios en línea, 9. Realizo trabajos grupales para desarrollar investigación con TIC, 10. El uso de TIC desarrolla habilidades propias de las ciencias naturales (comprender texto científico, explicar fenómenos e indagar). Esta escala tiene una fiabilidad alta (Alfa de Cronbach 0,791). La escala oscila entre cuatro valores de respuesta (0: nunca, 1: algunas veces, 2: muchas veces, 3 : siempre).

- Competencia cientifica (autopercibida). Esta escala de 10 ítems tiene una alta fiabilidad, con un alfa de Cronbach de 0,819. Contiene tres subescalas (uso comprensivo del conocimiento científico, explicación de fenómenos e indagación), que se basan en lo planteado por el Instituto Colombiano para la Evaluación de la Educación (2016), que describe la prueba de ciencias naturales para el grado 9, y se emplean las mismas modalidades de respuesta que en la escala anterior (0-4). La subescala de uso comprensivo del conocimiento incorpora ítems como «Observo, diferencio y describo objetos, eventos o fenómenos», «Identifico en la gráfica dos o más variables que describen un evento, organizo información relevante para responder 
una pregunta y acudo a libros u otras fuentes de información para resolver situaciones científicas» y tiene una fiabilidad moderada, con un alfa de Cronbach de 0,584. La subescala de explicación de fenómenos contiene ítems como "Creo argumentos lógicos de los fenómenos percibidos, confronto los resultados obtenidos en una investigación con mis propias hipótesis, formulo conclusiones a partir de la indagación» y tiene una fiabilidad moderada, con un alfa de Cronbach de 0,645. La subescala de indagación contiene ítems como «Formulo preguntas sobre eventos o fenómenos, planteo procedimientos para comprobar hipótesis o dar posibles soluciones a problemas científicos y diseño gráficas a partir de la información recogida» y tiene una fiabilidad alta, con un alfa de Cronbach de 0,902.

- Competencia científica observada en prueba de desempeño con actividades de ciencias naturales. Esta prueba está basada en PISA (2015), MEN (2013) y SABER ICFES (2016). Mide el desempeño y la competencia científica a través de 20 cuestiones de opción múltiple que recogen sendos casos y problemas. Por ejemplo: «Cuando Juan pasa por una zona donde el aire contiene gases tóxicos usa una máscara de gas que los filtra, como se muestra en la siguiente imagen, a partir de lo anterior, se puede afirmar que el aire contaminado...». Los resultados se evalúan como grado de desempeño: bajo (0-10), moderado (15-30), satisfactorio (35-40) y avanzado (45-50) (basados en PISA, 2015).

\subsection{Procedimiento de recogida de datos}

Los datos se recogieron a través del formulario creado en Google Docs con las escalas mencionadas y las preguntas de perfil. Se aplicó a través de la plataforma Edmodo a los estudiantes de octavo y noveno de educación básica secundaria en enero de 2019 y se preservó la identidad y el anonimato en las respuestas. Así mismo, la investigación se realizó con la autorización del comité de ética institucional previo informe favorable.

\subsection{Procedimiento de análisis de datos}

Tienen lugar pruebas de validación de constructo y de fiabilidad usando un análisis de componentes principales categóricos para establecer la conveniencia de las medidas empleadas en la muestra participante. Tanto la escala de aprendizaje con tecnologías digitales como la de competencia científica autopercibida presentan unidimensionalidad. Para el cálculo de las variables globales de cada escala se realiza una transformación de las variables partiendo del sumatorio de los ítems para construir una medida global de la experiencia de aprendizaje con tecnologías digitales y de la competencia científica autopercibida.

El análisis de datos que se sigue para dar cobertura al objetivo general es de tipo descriptivo y comparativo según la variable género. Se emplean medidas de tendencia central y de variabilidad para la exploración de variables y su distribución. Antes de proceder a la comparación de medias en las variables 
de interés respecto al género se realiza la prueba de Kolmogorov Smirnov, cuyos resultados denotan que estamos ante una distribución que no sigue la curva normal, y de Levene, que indica que existe homogeneidad de varianzas en los grupos de chicos y chicas, y el nivel de significación presenta valores mayores a $0,05(F=0,27, p=0,604)$. Partiendo de estos resultados, se realizan pruebas no paramétricas, concretamente se aplica la $U$ de Mann-Whitney para establecer si existen diferencias significativas en las variables consideradas según el género. Se realizan análisis de regresión lineal múltiple para determinar si existe efecto tanto de la experiencia de aprendizaje con tecnologías digitales como del desempeño en actividades de ciencias naturales sobre las competencias científicas autopercibidas. Para todo ello se emplea el programa informático SPSS, v. 26.

\section{Resultados}

\subsection{Experiencia de aprendizaje con tecnologias digitales de chicos y chicas}

En relación con el primer objetivo específico sobre conocer la experiencia de aprendizaje con tecnologías digitales que tiene el alumnado, los resultados muestran una incidencia baja con $\mathrm{M}=13,4, \mathrm{DT}=3,89(N=160$, Mín. = 5, Máx. $=23)$, teniendo en cuenta que la puntuación de la escala resultante oscila entre 0 y 30 (ver la figura 1 ).

Aunque la experiencia de aprendizaje con tecnologías digitales se observa baja, la exploración de cada uno de los ítems de la escala nos muestra que el estudiantado reconoce "manejar la computadora y navegar por internet sin muchas dificultades». Este ítem alcanza la media más elevada $(M=2,09$,

Figura 1. Experiencia de aprendizaje con tecnologías digitales

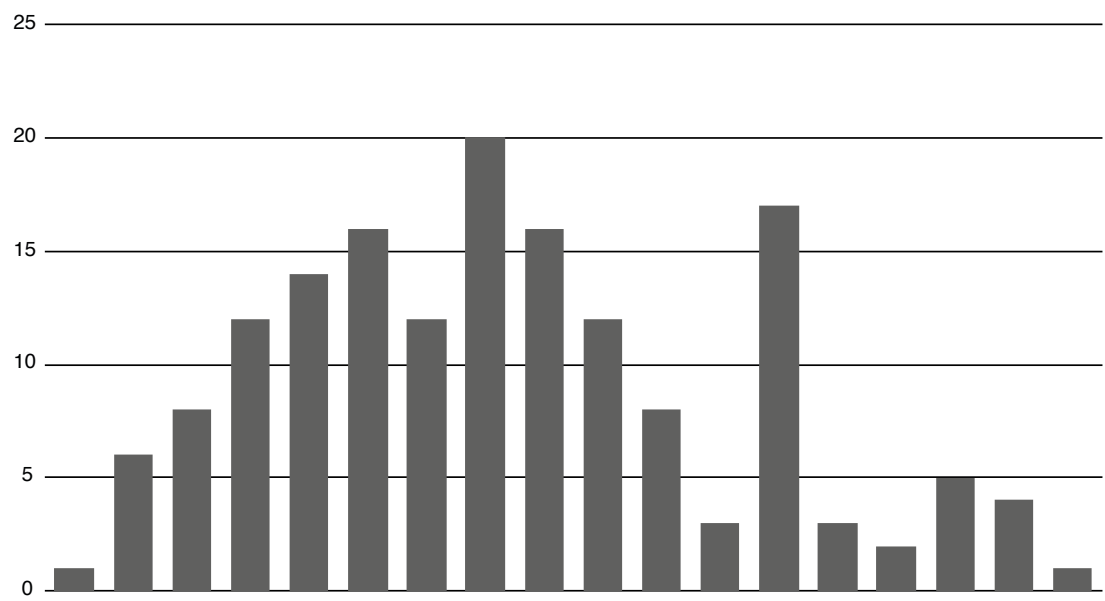

Fuente: elaboración propia. 
DT $=0,804)$ seguido de la idea de que «el uso de las TIC desarrolla habilidades propias de las Ciencias Naturales» $(\mathrm{M}=1,69$, DT = 0,69).

Los resultados exploratorios de la variable sobre experiencia de aprendizaje con tecnologías digitales según género $(\mathrm{M}$ (chicos) $=13,91$, DT (chicos) $=4,427 ; \mathrm{M}$ (chicas) $=13,65$, DT $($ Chicas $)=4,317)$ y la prueba U de MannWhitney $(\mathrm{U}=2900,500, p=0,305)$ indican que no hay diferencias significativas en la experiencia de aprendizaje con tecnologías digitales entre chicos y chicas.

\subsection{Competencia científica autopercibida según el género}

En relación con el segundo objetivo relacionado con conocer las competencias científicas autopercibidas diferenciando por género, los resultados muestran que la competencia científica autopercibida muestra una tendencia baja con $\mathrm{M}=13,78$ y DT = 4,36, $(N=160$, Mín. = 3, Máx. = 24) lo que denota amplia dispersión en las respuestas dadas (ver figura 2).

Los resultados muestran que no hay diferencias significativas entre chicos y chicas en la competencia científica autopercibida, ni en ninguna de sus dimensiones consideradas, como se puede apreciar en la tabla 1 .

Figura 2. Competencia científica autopercibida según el género

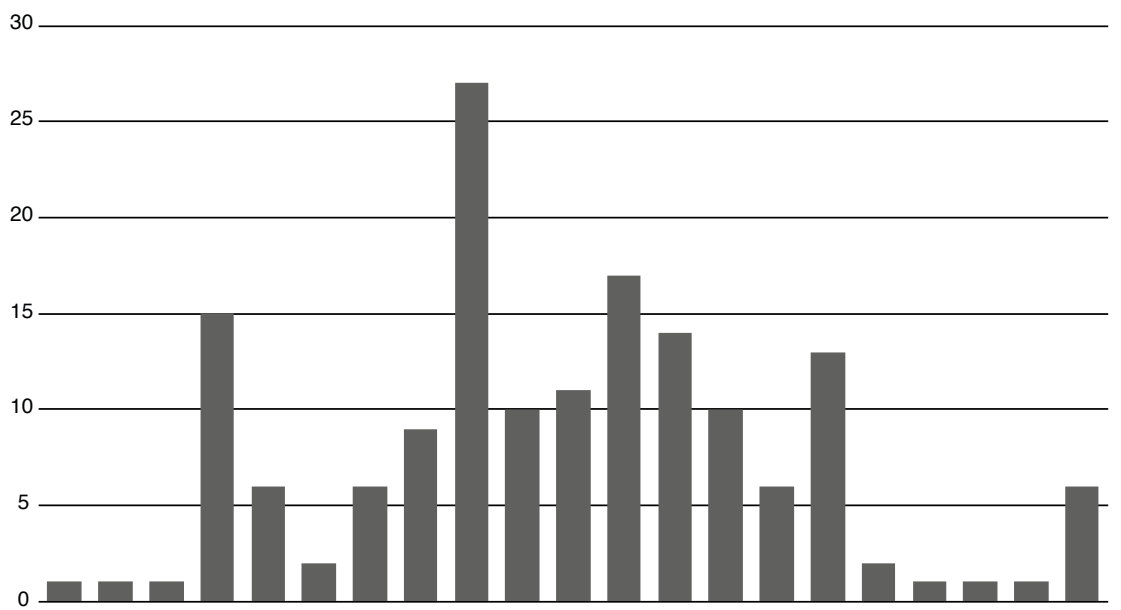

Fuente: elaboración propia. 
Tabla 1. $U$ de Mann-Whitney para competencia científica autopercibida por género

\begin{tabular}{llllll}
\hline U de Mann-Whitney & Género & $\boldsymbol{n}$ & Rangos & $\mathbf{U}$ & $\boldsymbol{p}$ \\
\hline Competencia científica autopercibida & & & & & \\
Uso comprensivo del conocimiento científico & Chicas & 80 & 83,06 & 2995,000 & 0,478 \\
& Chicos & 80 & 77,94 & & \\
\hline Explicación de fenómenos & Chicas & 80 & 74,44 & 2715,500 & 0,092 \\
& Chicos & 80 & 86,56 & & \\
\hline Indagación & Chicas & 80 & 75,35 & 2788,000 & 0,150 \\
& Chicos & 80 & 85,65 & & \\
Competencia científica global & Chicas & 80 & 78,64 & 3050,000 & 0,611 \\
& Chicos & 80 & 82,36 & & \\
\hline
\end{tabular}

Fuente: elaboración propia.

\subsection{Competencia cientifica observada en el desempeño de actividades de ciencias naturales según el género}

En relación con el objetivo específico de conocer las competencias científicas observadas a partir del desempeño del alumnado en actividades de ciencias naturales relacionadas con contenidos sobre entorno vivo; entorno físico, y ciencia, tecnología y sociedad, los resultados indican que el nivel de desempeño que reúne un mayor porcentaje es el moderado, tanto en las actividades sobre entorno vivo (un 45,6\%) como en las actividades sobre entorno físico (un 53,8\%) y en las de ciencia, tecnología y sociedad (un 64,4\%). Los desempeños avanzados se alcanzan en un $4,4 \%$ en actividades sobre entorno físico y en un 2,5\% en actividades sobre ciencia, tecnología y sociedad. En las actividades sobre entorno vivo no se aprecia el desempeño avanzado (ver tabla 2).

El coeficiente de contingencia muestra que no hay diferencias significativas en función del género ni en el desempeño en las actividades sobre entorno vivo $(\mathrm{C}=0,044, p=0,857)$, ni en las actividades sobre entorno físico $(\mathrm{C}=0,123, p=0,486)$, ni en las de ciencia, tecnología y sociedad $(\mathrm{C}=0,129, p=0,436)$. 
Tabla 2. Descripción en porcentaje de niveles de desempeño por género

\begin{tabular}{|c|c|c|c|c|c|}
\hline $\begin{array}{l}\text { Competencias } \\
\text { científicas en } \\
\text { actividades } \\
\text { mediadas por } \\
\text { tecnologías } \\
\text { digitales }\end{array}$ & Género & $\begin{array}{c}\% \\
\text { desempeño } \\
\text { bajo }\end{array}$ & $\begin{array}{c}\% \\
\text { desempeño } \\
\text { moderado }\end{array}$ & $\begin{array}{c}\% \\
\text { desempeño } \\
\text { satisfactorio }\end{array}$ & $\begin{array}{c}\% \\
\text { desempeño } \\
\text { avanzado }\end{array}$ \\
\hline \multirow{2}{*}{$\begin{array}{l}\text { Actividades sobre } \\
\text { entorno vivo }\end{array}$} & Chicas & 35,0 & 46,3 & 18,8 & \\
\hline & Chicos & 38,8 & 45,0 & 16,3 & \\
\hline Total & & 36,9 & 45,6 & 17,5 & \\
\hline \multirow{2}{*}{$\begin{array}{l}\text { Actividades sobre } \\
\text { entorno físico }\end{array}$} & Chicas & 22,5 & 56,3 & 15,0 & 6,3 \\
\hline & Chicos & 25,0 & 51,2 & 21,3 & 2,5 \\
\hline Total & & 23,8 & 53,8 & 18,1 & 4,4 \\
\hline \multirow{3}{*}{$\begin{array}{l}\text { Desempeño en } \\
\text { ciencia, tecnología y } \\
\text { sociedad }\end{array}$} & Chicas & 7,5 & 65,0 & 25,0 & 2,5 \\
\hline & Chicos & 15,0 & 63,7 & 18,8 & 2,5 \\
\hline & & 11,3 & 64,4 & 21,9 & 2,5 \\
\hline
\end{tabular}

Fuente: elaboración propia.

\subsection{Variables predictoras de la competencia cientifica autopercibida según el género}

Para conocer si la experiencia de aprendizaje con tecnologías digitales y el desempeño en actividades de ciencias naturales predice la autopercepción acerca del uso comprensivo del conocimiento (como dimensión de la competencia científica autopercibida), se lleva a cabo una regresión lineal múltiple. Los resultados del modelo indican que la experiencia de aprendizaje con tecnologías digitales predice la variable dependiente en un 17,2\%. Los indicadores de multicolinealidad VIF (Variance Inflation Factor) inferiores a 10 y los valores de tolerancia mayores a 0,20 indican que no existen correlaciones elevadas entre los factores del modelo, el indicador de Durbin Watson presenta un valor aceptable para la generalización de los datos $(1,658)$.

Para determinar si la experiencia de aprendizaje con tecnologías digitales y el desempeño en actividades de ciencias naturales predice la autopercepción de la explicación de los fenómenos, se lleva a cabo una regresión lineal. Los resultados del modelo indican que los aprendizajes de tecnologías digitales predicen la variable dependiente en un $25,2 \%$. Los indicadores de multicolinealidad VIF (Variance Inflation Factor) inferiores a $10(1,005-1,118)$ y los valores de tolerancia que están entre 0,993 y 0,995 indican que no existen correlaciones elevadas entre los factores del modelo, y el indicador de Durbin Watson presenta un valor aceptable para la generalización de los datos $(1,726)$.

Para determinar si la experiencia de aprendizaje con tecnologías digitales y el desempeño en actividades de ciencias naturales predicen la autopercepción sobre la indagación, se lleva a cabo una regresión lineal. Los resultados del modelo indican que la experiencia de aprendizaje con tecnologías digitales 
predice la variable dependiente en un 26,3\%. Los indicadores de multicolinealidad VIF y los valores de tolerancia que están son iguales que en los dos modelos anteriores e indican que no existen correlaciones elevadas entre los factores del modelo, y el indicador de Durbin Watson es de 1,641 (ver tabla 3).

Los resultados de los modelos de regresión indican que la experiencia de aprendizaje con tecnologías digitales predice el uso comprensivo del conocimiento científico, la explicación de fenómenos científicos y la indagación, es decir, la competencia científica autopercibida.

Tabla 3. Modelos de regresión para competencias científicas autopercibidas

\begin{tabular}{|c|c|c|c|c|c|c|c|c|}
\hline Modelos & $\mathbf{F}$ & $\mathbf{R}^{2}$ & $\Delta \mathbf{R}^{2}$ & B & $\begin{array}{l}\text { Error } \\
\text { estándar }\end{array}$ & B & $p$ & $1-\beta$ \\
\hline $\begin{array}{l}\text { Modelo 1. Uso compresivo del } \\
\text { conocimiento }\end{array}$ & $\begin{array}{l}8.041 \\
(4,155)\end{array}$ & 0,172 & 0,150 & & & & 0,001 & 0,99 \\
\hline (Constante) & & & & 0,898 & 0,184 & & 0,001 & \\
\hline Actividades sobre entorno vivo & & & & 0,014 & 0,037 & 0,029 & 0,697 & \\
\hline Actividades sobre entorno físico & & & & 0,035 & 0,029 & 0,095 & 0,223 & \\
\hline Ciencia, tecnología y sociedad & & & & $-0,035$ & 0,035 & $-0,075$ & 0,325 & \\
\hline $\begin{array}{l}\text { Aprendizaje con tecnologías } \\
\text { digitales }\end{array}$ & & & & 0,484 & 0,090 & 0,394 & 0,001 & \\
\hline $\begin{array}{l}\text { Modelo 2. Explicación de los } \\
\text { fenómenos }\end{array}$ & $\begin{array}{l}13.055 \\
(4,155)\end{array}$ & 0,252 & 0,233 & & & & 0,001 & 0,99 \\
\hline (Constante) & & & & 0,274 & 0,204 & & 0,182 & \\
\hline Actividades sobre entorno vivo & & & & $-0,037$ & 0,041 & $-0,064$ & 0,375 & \\
\hline Actividades sobre entorno físico & & & & 0,053 & 0,032 & 0,123 & 0,097 & \\
\hline Ciencia, tecnología y sociedad & & & & 0,022 & 0,039 & 0,040 & 0,578 & \\
\hline $\begin{array}{l}\text { Aprendizaje con tecnologías } \\
\text { digitales }\end{array}$ & & & & 0,685 & 0,1 & 0,475 & 0,001 & \\
\hline Modelo 3. Indagación & $\begin{array}{l}13.806 \\
(4,155)\end{array}$ & 0,263 & 0,244 & & & & 0,001 & 0,99 \\
\hline (Constante) & & & & 0,354 & 0,199 & & 0,078 & \\
\hline Actividades sobre entorno vivo & & & & $-0,052$ & 0,040 & $-0,092$ & 0,196 & \\
\hline Actividades sobre entorno físico & & & & 0,057 & 0,031 & 0,133 & 0,070 & \\
\hline Ciencia, tecnología y sociedad & & & & $-0,022$ & 0,038 & $-0,042$ & 0,562 & \\
\hline $\begin{array}{l}\text { Aprendizaje con tecnologías } \\
\text { digitales }\end{array}$ & & & & 0,682 & 0,098 & 0,482 & 0,001 & \\
\hline
\end{tabular}

Fuente: elaboración propia. 


\section{Discusión de resultados y conclusiones}

En la educación básica resulta importante ofrecer a todos los estudiantes aprendizajes de calidad y garantizar la llegada de nuevas generaciones de científicos y científicas a la sociedad. El hecho de que las tecnologías puedan ser un factor promotor de la capacitación científica es el principal interés de fondo en este trabajo. La finalidad última de plantear propuestas didácticas apoyadas en tecnologías digitales para la enseñanza de las ciencias que nos permitan desarrollar las competencias científicas es un importante acicate para el mundo educativo y para quienes nos dedicamos a la enseñanza. Sin embargo, esta investigación ha desvelado el poder predictor de la experiencia de aprendizaje con tecnologías digitales en la competencia científica (autopercibida). Con ello, hemos podido mostrar un beneficio potencial de las tecnologías digitales en la línea que planteaban Petko et al. (2017), quienes apuntaban a la hipótesis de que la experiencia con tecnologías digitales y la calidad de uso sea más importante, en términos de aprendizaje, que la cantidad de uso. No obstante, se requiere mayor investigación que nos permita, de acuerdo con Hu et al. (2018), mejorar la calidad de la integración de las tecnologías digitales en entornos educativos y vincularlas a acciones que garanticen un impacto positivo en las competencias científicas. También el estudio pone de manifiesto la influencia entre ambas variables, al igual que los trabajos que mostraban en su recopilación Romero y Quesada (2014) o el aporte de Luu y Freeman (2011) sobre el impacto de las tecnologías digitales en el logro en materias relacionadas con ciencia.

Los resultados obtenidos en este trabajo están próximos a resultados de investigaciones que relacionan la percepción sobre el uso de las tecnologías digitales para aprender con una mejora del rendimiento en ciencias (Petko et al., 2017). El estudio de Hu et al. (2018) incorpora algunos aspectos relacionados con la experiencia de aprendizaje con tecnologías digitales como pueden ser la conectividad o el número de ordenadores en función del tamaño del centro. Estos aspectos también han sido considerados en el presente trabajo. No obstante, los autores no encuentran relacionados estos indicadores con la alfabetización científica. Esto puede suponer una limitación del estudio al incorporarlos en la medida de la experiencia, lo que lleva a poder plantear una revisión de estos indicadores en la medida de la experiencia. Además, también es coincidente con estudios sobre el impacto.

En cuanto a la competencia científica autopercibida, los resultados muestran una tendencia a la baja, y en cuanto a la competencia científica observada, el desempeño es moderado. Los análisis según género ponen de manifiesto que no hay diferencias significativas entre chicos y chicas en ninguna de las áreas de contenido consideradas. Este dato es coincidente con estudios como el de Naganuma (2017) y el de Hardinata et al. (2019), que revelan que no hay diferencias significativas entre chicos y chicas en la alfabetización científica a nivel de educación secundaria. Sin embargo, contradice estudios como el de Nugraheni (2020) o el de Kristyasari et al. (2018), que demuestran que existe una diferencia significativa entre los puntajes promedio de alfabetización científica de las 
estudiantes mujeres con respecto a los estudiantes hombres. En este estudio de Nugraheni (2020) el puntaje promedio de la alfabetización científica de las estudiantes fue mayor que el puntaje promedio de los hombres.

Algunas implicaciones de este estudio están relacionadas con los esfuerzos actuales en la formación básica de las chicas para que accedan a las carreras tecnológicas, dadas las brechas en el ámbito STEM. A pesar de que los estudios en secundaria muestran que no hay diferencias en el desempeño (Naganuma, 2017) y si las hay ellas presentan mayor nivel (Nugraheni, 2020), las experiencias didácticas que se planteen en secundaria deben ser sensibles a un enfoque de género y valorar y evaluar impactos para una enseñanza eficaz.

\section{Referencias bibliográficas}

Hardinata, A.; Putri, R.E. y Permanasari, A. (2019). Gender difference and scientific literacy level of secondary student: A study on global warming theme. Journal of Physics: Conference Series, 1157(2), 022016.

Hillmayr, D.; Ziernwald, L.; Reinhold, F.; Hofer, S.I. y Reiss, K.M. (2020). The potential of digital tools to enhance mathematics and science learning in secondary schools: A context-specific meta-analysis. Computers \& Education, 153, 103897.

Hu, X.; Gong, Y.; LaI, Ch. y Leung, F. (2018). The relationship between ICT and student literacy in mathematics, reading, and science across 44 countries: A multilevel analisis. Computers \& Education, 125, 1-13.

Instituto Colombiano para la Evaluación de la Educación (ICFES) (2016). Saber $9^{\circ}$ : Lineamientos para la aplicación muestral 2016. Recuperado de <www. icfes.gov.co/docman/...y.../pruebas-saber...9-lineamientos...2016/file>.

KolB, A.Y. y Kolb, D.A. (2017). Experiential learning theory as a guide for experiential educators in higher education. Experiential Learning \& Teaching in Higher Education, 1(1), 7-44.

Kristyasari, M.L.; Yamtinah, S.; Utomo, S.B. e Indriyanti, N.Y. (2018). Gender differences in students' science literacy towards learning on integrated science subject. Journal of Physics: Conference Series, 1097(1), 012002.

Luu, K. y Freeman, J. (2011). An analysis of the relationship between information and communication technology (ICT) and scientific literacy in Canada and Australia. Computers \& Education, 56(4), 1072-1082.

MEN (2004). Estándares Básicos de Competencias en Ciencias Naturales y Ciencias Sociales. Formar en ciencias: ;El desafio! Lo que necesitamos saber y saber hacer. Recuperado de <https://www.mineducacion.gov.co/1759/articles-81033_archivo_pdf.pdf>.

- (2013). Estándares Básicos de Competencias en Ciencias Sociales y Ciencias Naturales. Recuperado de <https://www.mineducacion.gov.co/1621/articles-116042_archivo_ pdf3.pdfs.

- (2017). Derechos Básicos de Aprendizaje-DBA: Ciencias Naturales. Recuperado de $<$ http://aprende.colombiaaprende.edu.co/sites/default/files/naspublic/DBA_C. Naturales.pdf $>$.

Naganuma, S. (2017). An assessment of civic scientific literacy in Japan: Development of a more authentic assessment task and scoring rubric. International Journal of Science Education, 7(4), 301-322.

Neiman, A. (2017). El aula extendida y el uso de tecnología digital: Habilidades cognitivas implicadas en contenidos de Biología. Tesis de maestría. Buenos Aires, Argentina. Universidad Nacional de La Plata. $<$ https://doi.org/10.35537/10915/62524> 
Nugraheni, N.C. (2020). Scientific literacy profile of X grade students in Gunungkidul on Biology reviewed based on gender. Journal of Physics: Conference Series, 1440(1), 012070.

OCDE (2015). The ABC of Gender Equality in Education: Aptitude, Behaviour, Confidence. París. OECD Publishing.

- (2017). PISA 2015 technical report. París. OECD Publishing.

Odell, B.; Galovan, A.M. y Cutumisu, M. (2020). The Relation between ICT and Science in PISA 2015 for Bulgarian and Finnish Students. Eurasia Journal of Mathematics, Science and Technology Education, 16(6), em1846. <https://doi.org/10.29333/ejmste/7805>

Parno, P.; Yuliati, L.; Hermanto, F.M. y Ali, M. (2020). A Case Study on Comparison of High School Students' Scientific Literacy Competencies Domain in Physics with Different Methods: Pbl-Stem Education, Pbl, and Conventional Learning. Journal Pendidikan IPA Indonesia, 9(2), 159-168.

PÉrez VAlverde, J. (2015). El uso de las TIC y su incidencia en el interaprendizaje en el área de las ciencias naturales de los estudiantes del séptimo grado de educación general básica de la escuela "Nicolás Martínez» de la parroquia San Bartolomé de Pinllo del Cantón Ambato, de la provincia del Tungurahua. Tesis de pregrado. Ecuador. Universidad Técnica de Ambato.

Petko, D.; Cantieni, A. y Prasse, D. (2017). Perceived quality of educational technology matters a secondary analysis of students' ICT use, ICT-related attitudes, and PISA 2012 test scores. Journal of Educational Computing Research, 54(8), 1070-1091.

RamíreZ, J.M. y DÁvila, M.A. (2017). Las emociones según el género, en el aprendizaje de la Tecnología del alumnado de primer curso de Educación Secundaria Obligatoria. Apice: Revista de Educación Científica, 1(2), 18-37. <https://doi.org/10.17979/ arec.2017.1.2.2091>

Rincón, F. (2015). Tendencias sobre el uso de TIC en la enseñanza de las ciencias a la luz de las revistas: Computers \& Education y Enseñanza de las Ciencias. Tesis de maestría. Bogotá, Colombia. Universidad Distrital Francisco José de Caldas. Recuperado de <http://repository.udistrital.edu.co/handle/11349/2160>.

Romero, M. y QuesadA, A. (2014). Nuevas tecnologías y aprendizaje significativo de las ciencias. Enseñanza de las Ciencias, 32(1), 101-115.

Rosa, G.C.; Cari, C.; Aminah, N.S. y Handhika, J. (2018). Students' understanding level and scientific literacy competencies related to momentum and impulse. Journal of Physics: Conference Series, 1, 012019.

SidDiQ, F. y Scherer, R. (2019). Is there a gender gap?: A meta-analysis of the gender differences in students' ICT literacy. Educational Research Review, 27, 205-217.

TsaI, Ch.Y. (2018). The effect of online argumentation of socio-scientific issues on students' scientific competencies and sustainability attitudes. Computers \& Education, 116, 14-16.

Voyer, S. y Voyer, D. (2014). Gender differences in scholastic achievement: A metaanalysis. Psychological Bulletin, 140(4), 1174-1204. <https://doi.org/10.1037/a0036620>

Winarni, E.W.; Hambali, D. y Purwandari, E.P. (2020). Analysis of Language and Scientific Literacy Skills for 4th Grade Elementary School Students through Discovery Learning and ICT Media. International Journal of Instruction, 13(2), 213-222. <https://doi.org/10.29333/iji.2020.13215a> 